\title{
Implementation via rights structures
}

\author{
Semih Koray, Kemal Yildiz* \\ Bilkent University, Department of Economics, Turkey \\ Received 8 October 2015; final version received 16 November 2017; accepted 16 April 2018 \\ Available online 24 April 2018
}

\begin{abstract}
Implementation of socially desirable alternatives can be thought of as a way to design power distribution in a society such that the equilibrium outcomes coincide with the alternatives chosen at each preference profile. In this paper, we introduce a new institutional framework for implementation, which takes power distribution in a society as its point of departure. We use the notion of a rights structure, introduced by Sertel (2001), to formalize the power distribution in a society. We formulate and characterize implementability via rights structures under different specifications, which require having well-defined convergence dynamics and being consistent with farsighted behavior. We identify how implementation via rights structures is related to implementation via mechanisms. In the presence of at least three agents, we find the class of rights structures, implementability via which is equivalent to Nash and strong Nash implementability. We also introduce a strategic counterpart of implementation via rights structures in terms of deviation-constrained mechanisms.
\end{abstract}

(c) 2018 Elsevier Inc. All rights reserved.

JEL classification: D01; D02; D71

Keywords: Implementation; Rights structures; Nash equilibrium; Monotonicity; Social choice rule

\footnotetext{
the are grateful to Ariel Rubinstein for many long conversations, insightful suggestions and encouragement. We thank the associate editor and an anonymous referee for their helpful comments. We also thank Cagri Akkoyun, Hasan T. Apakan, Kivanc Akoz, Geoffroy de Clippel, Battal Dogan, Jon Eguia, Kfir Eliaz, Itzhak Gilboa, Faruk Gul, Johannes Horner, Tarik Kara, Herve Moulin, Efe Ok, David Pearce, Michael Richter, Rani Spiegler, Andrea Wilson, and Charles Wilson, seminar participants at Tel Aviv University, NYU, University of St. Andrews, Bilkent University, Yeshiva University, Bogazici University, Sabanci University, 2013 North American Summer Meeting of Econometric Society, and Bosphorus Workshop on Economic Design for their helpful comments. We thank Christopher Eyer for his linguistic review of the paper. Kemal Yildiz gratefully acknowledges the support of European Research Council Fellowship.

$*$ Corresponding author.

E-mail addresses: ksemih@bilkent.edu.tr (S. Koray),kemal.yildiz@bilkent.edu.tr (K. Yildiz).
} 


\section{Introduction}

It is hard to deny the steadily growing role of implementation in economic theory over the past four decades. Every modern society is familiar with institutional "real life" mechanisms, such as constitutions, legal codes, rules of corporate culture and social norms that aim to rule out socially undesirable outcomes and implement solely desirable ones under different circumstances. Such real life mechanisms, however, often have fuzzy objective with questionable social desirability. The constructs that link the objective to the existing societal preference profile are usually rough. Even in economic regulation, which may be regarded as a field of relatively well-posed problems in contrast to other areas, mechanism design was conducted more or less by "trial and error" until the 1970s, as the objective of the mechanism was not precisely defined.

Implementation theory takes the social objective-mostly represented by a social choice rule-as given. Thus, its point of departure is a precisely defined object, which the society is assumed to have somehow unanimously agreed upon. Moreover, the objective relates the social desirability of an outcome to the current societal preference profile and other relevant parameters in a precise manner. It is exactly this ambitious aim that gives rise to the main problem concerning implementation, as the designer lacks the ability to observe the individuals' actual preferences. Beginning with Hurwicz (1972), the notion of a game form has been introduced to deal with this problem.

A game form - together with a game-theoretic equilibrium notion-is used to implement a social choice rule. The selected equilibrium notion is assumed to reflect the behavioral fundaments of the individuals in the society. In the last three decades, many studies have been successful in identifying implementable social choice rules according to widely used equilibrium notions. ${ }^{1}$ However, a persistent criticism of implementation theory has been that the game forms constructed to obtain general results have "unnatural" features that take away from the relevance of the theory. ${ }^{2}$ Jackson (1992) and Abreu and Matsushima (1992) argue in detail besides being difficult to interpret, there are several technical problems associated with these games.

In our view, the main question is whether the framework within which the agents interact is sufficiently familiar and understandable, so that the agents will act in predictable ways. Thus, the introduction of a framework for implementation, formulated in a language closer to that of real life mechanisms, might be useful in dealing with this problem. One way of describing what implementation via a mechanism does is that the conjunction of a game form with an equilibrium notion results in a power distribution ${ }^{3}$ in the society under which the equilibrium outcomes coincide with those of the social choice rule to be implemented. A natural question that then arises is whether or not the power distribution that does the job can be taken as the point of departure in implementation. This is the main problem that we deal with in this paper.

From among several possibilities to represent a power distribution, the notion of a rights structure introduced by Sertel (2001) seems to be the best fit for our approach. Similar to the

\footnotetext{
1 For instance see Abreu and Sen (1991), Moore and Repullo (1988), Danilov (1992), Dutta and Sen (1991, 2012), Palfrey and Srivastava (1991), McKelvey (1989).

2 Specifically, some sort of integer game or modulo game is used to eliminate strategies with unacceptable outcomes from the equilibria. In these games, whenever there is no consensus, the agent who announces the highest integer gets to be a dictator. We use a similar construction in the proof of Proposition 3 to establish a connection between our model and classical implementation.

3 Moulin and Peleg (1982) formally proposed the notion of an effectivity function to describe the power distribution induced from a mechanism. We discuss how this notion is carried to the implementation problem while situating our work within the existing literature.
} 
classical setup, we have a society and a set of alternatives, over which our society is endowed with a preference profile. A rights structure consists of a state space $S$, an outcome function $h$, which associates an alternative with each state, and a code of rights $\gamma$. A code of rights $\gamma$ specifies the power distribution in our society by assigning to each pair of distinct states $(s, t)$ the family $\gamma(s, t)$ of coalitions that are entitled to approve a change from state $s$ to state $t$. Thus, an existing state $s$ will be converted to another state $t$ only if there is some coalition which is entitled to change $s$ to $t$ and is willing to do so. In the absence of a state $t$ different than $s$, along with such a coalition, it is only natural to regard $s$ as an equilibrium state under the given rights structure and the society's present preference profile. Moreover, a social choice rule is said to be implemented by the rights structure in question, if for each preference profile, alternatives chosen by the social choice rule coincide with the equilibrium outcomes that are induced by the equilibrium states via the outcome function $h$.

The major job accomplished by a rights structure can be considered on two different grounds. One is that it provides an alternative way of implementing a social choice rule, in which the required decision process on the part of the agents is rather transparent. The main formulas of both classical implementation and implementation via rights structure reflect the simple wisdom that "those who wish are not able, while those who are able do not wish". To put this wisdom into practice via the classical approach, each agent needs to consider each joint strategy and consider how each other agent can affect the outcome by unilaterally changing his strategy. In implementation via a rights structure, however, the "main formula" of implementation is made visible with naked eye. In that, all we expect of the agents is that they compare the incumbent state with others through the alternatives they induce and change the status quo into some other state in case they wish and are entitled to do so. The second accomplishment of a rights structure is that it sheds light on the rights structures underlying implementation via a mechanism in different equilibrium notions.

The simplicity of the construct we have introduced is also reflected in our characterization results. A social choice rule turns out to be implementable via a rights structure if and only if it is image monotonic, which gets reduced to Maskin monotonicity for unanimous social choice rules. In addition, any social choice rule that is implementable via a rights structure is also implementable in an individual-based manner. This means that any nonempty collection of coalitions entitled to approve a change from a state into another one can be taken to consist of singletons only. As an important special case, when we take the state space to be equal to the set of alternatives, implementability via rights structures is observed to be equivalent to Maskin monotonicity strengthened by an additional property that we call binary consistency.

One possible reason for the simplicity and generality of implementation via a rights structure is that our framework leaves unspecified the details of agents' interaction in reaching an equilibrium. In this study, we addressed this problem on two different grounds, one of which is concerned with convergence from a nonequilibrium state into an equilibrium state. For any unanimous social choice rule $F$ that is implementable via a rights structure, it is possible to design the rights structure used to implement $F$ in such a way that each equilibrium state can be reached from any nonequilibrium state via a sequence of at most two states such that the passage from each state in the sequence to the next one is justified in regards to both rights and preferences. ${ }^{4}$ We also characterize implementability of a social choice rule via a rights structure when our

\footnotetext{
4 This is in the vein of recurrent implementation proposed by Cabrales and Serrano (2011), which we discuss at the end of Section 4.1.
} 
agents are farsighted rather than myopic, in the sense that, in changing a state into another one, they make sure that the latter state is an equilibrium itself and thus cannot be changed further into a third state.

The second ground on which we deal with this problem is that we find the precise strategic counterpart of implementability via rights structures in terms of mechanisms. In a classical mechanism, each agent is allowed to deviate from any joint strategy by choosing any strategy whatsoever from her strategy space. In a deviation-constrained mechanism, on the other hand, the admissible deviations from each joint strategy are prespecified for each agent. ${ }^{5}$ We show that implementability via a deviation-constrained mechanism and implementability via a rights structure coincide in the presence of at least three agents.

The notion of implementation via rights structures also yields novel characterizations of Nash and strong Nash implementability via a mechanism. The common attributes of a rights structure that distinguish Nash-implementable social choice rules in the presence of at least three agents are shown to be individual-basedness and individual-transitivity. As every social choice rule that is implementable via a rights structure is also implementable via an individual based rights structure, the distinctive feature of the rights structures underlying Nash implementability turns out to be individual-transitivity.

The rest of the paper is organized as follows. Section 2 is concerned with the related literature, then we introduce the formal model along with several examples in Section 3. In Section 4, we give a characterization of social choice rules implementable via a rights structure, and also deal with convergence dynamics and farsighted implementability. In Section 5, novel characterizations of Nash and strong Nash implementability are given in terms of rights structures. Section 6 deals with the particular case, where the state space coincides with the set of alternatives. Section 7 introduces a strategic counterpart of implementation via rights structures in terms of deviation-constrained mechanisms. Finally Section 8 concludes the paper.

\section{Relation to the literature}

The idea that rights should be incorporated into social choice theory originates from the seminal work of Sen (1970) on the impossibility of a Paretian liberal. Following Sen (1970), Moulin and Peleg (1982) proposed the notion of an effectivity function ${ }^{6}$ as a representation of the power distribution induced from a mechanism. Peleg and Winter (2002) then carried the notion of an effectivity function to the implementation framework. They argue that a certain mechanism implementing a social choice rule does not mean that this mechanism is the most desirable one for implementation. They formulate the so-called constitutional implementation, which requires the implementation of a given social choice rule via a mechanism that induces the same effectivity function as the social choice rule that is implemented. Here, we adopt a different approach to implementing a social choice rule-we propose explicitly designing a rights structure that represents the power distribution in a society.

\footnotetext{
5 There is wealth of research on mechanism design with evidence and mechanism design with boundedly rational agents. Our work closely aligns with Kartik and Tercieux (2012) and de Clippel (2014). We will compare and contrast our work with these studies in Section 7.

6 The idea dates back to Rosenthal (1972).
} 
The notion of a rights structure - the main tool we use for design - is a slightly simplified version of the Rechtsstaat, ${ }^{7}$ formulated by Sertel (2001). More recently, McQuillin and Sugden (2011) propose a similar notion, game in transition function form, as a generalization of effectivity functions. Both Sertel, and McQuillin and Sugden investigate the existence and efficiency of equilibria. In particular, Sertel renders counterparts of the first and second welfare theorems in this general framework. Since neither of these studies is in a design framework, none of their results have any implications for the results in this paper.

In another related study, Acemoglu et al. (2012) propose a framework similar to rights structures to study dynamic collective decision making problems. In this framework, a state determines a social situation as well as the coalitions that are entitled to move from the current state to the others. Conceptually, a rights structure is less restrictive, since the states from which a coalition is entitled to move can depend on the particular state that the coalition moves to. In their analysis, the authors focus on dynamic equilibria with sufficiently forward-looking agents, and characterize the set of dynamically stable states. In contrast, we are interested in designing rights structures to implement given social choice rules.

In Section 7, we provide a strategic counterpart of implementability via rights structures in terms of mechanisms. We compare and contrast implementability with deviation-constrained mechanisms with recent research on mechanism design with evidence ${ }^{8}$ and mechanism design with boundedly rational agents.

\section{Model}

We use $A$ to denote a nonempty, finite set of alternatives, and $N$ a nonempty finite set of agents. We denote the number of agents in the society by $n$. Each nonempty subset of $N$ is called a coalition, and denoted generically by $K$. Given $A$ and $N$, for each $i \in N, u_{i}$ denotes the preference relation - a complete, transitive, antisymmetric binary relation-on $A$ of agent $i$. For each distinct pair $a, b \in A, a u_{i} b$ denotes that " $i$ prefers $a$ to $b$ ". A preference profile $u=\left(u_{1}, \ldots, u_{n}\right)$. The collection of all preference profiles is denoted by $\mathcal{P}$. A social choice rule (SCR) $F$ maps each preference profile into a nonempty subset of $A$, i.e. $F: \mathcal{P} \rightarrow 2^{A} \backslash\{\emptyset\}$. An SCR $F$ is unanimous if for each $a \in A$ we have $F(u)=\{a\}$ whenever every agent in the society prefers $a$ to all other alternatives.

In classical implementation, the main design tool is a mechanism, which is a pair $(M, g)$. The joint strategy space is $M=\Pi_{i \in N} M_{i}$, where $M_{i}$ stands for the strategy set of agent $i$. The outcome function $g$ maps every joint strategy to an alternative, i.e. $g: M \rightarrow A$. A mechanism $(M, g)$ combined with a preference profile $u \in \mathcal{P}$, constitutes a normal form game. We denote the pure strategy Nash equilibria of this game by $\mathrm{NE}(M, g, u)$. An SCR $F$ is Nash-implementable via a mechanism $(M, g)$ if at each preference profile $u$, the alternatives chosen by $F$ coincide with the pure strategy Nash equilibrium outcomes of the game at given $u$, i.e. for each $u \in \mathcal{P}$, $F(u)=\{g(m): m \in N E(M, g, u)\}$.

\footnotetext{
$\overline{7}$ In addition to a rights structure, a Rechtsstaat contains an ability map, which associates each distinct state pair $(s, t)$ with a collection of coalitions, each of which is meant to be-cognitively or technologically—able to transform state $s$ into state $t$. This transformation requires the existence of two coalitions, one that is able to achieve this change and one that approves it.

8 Most of this literature concerns partial-implementation in the availability of verifiable evidence. An early reference is Green and Laffont (1986); more recent studies include Bull and Watson (2004, 2007), Kartik and Tercieux (2012), and Deneckere and Severinov (2008).
} 
In our framework, the main design tool is the rights structure $\Gamma$ introduced by Sertel (2001). A rights structure $\Gamma$ is a triple $(S, h, \gamma)$. We use $S$ to denote the state space, ${ }^{9}$ and $h$ the outcome function, which maps each state to an alternative, i.e. $h: S \rightarrow A$. Given a state space $S$, a code of rights specifies for each pair of distinct states $s, t \in S$, a family of coalitions denoted by $\gamma(s, t)$. We interpret that each coalition in $\gamma(s, t)$ is entitled to approve a change from $s$ to $t$ by the code of rights $\gamma$. For an additionally given outcome function $h$ and preference profile $u$, and for each distinct $s, t \in S$ a coalition $K$ prefers $t$ to $s$ if and only if for each $i \in K, h(t) u_{i} h(s)$. This is denoted by $h(t) u_{K} h(s)$. In order to define $\Gamma$-implementability, first we will specify the $\Gamma$-equilibrium notion, which plays the role of the solution concepts in the classical implementation.

Definition 1. Given a rights structure $\Gamma=(S, h, \gamma)$, and a preference profile $u \in \mathcal{P}$, a state $s \in S$ is a $\Gamma$-equilibrium at $u$ if for each $t \in S \backslash\{s\}$ there is no coalition $K \in \gamma(s, t)$ with $h(t) u_{K} h(s)$.

A state $s$ is a $\Gamma$-equilibrium at preference profile $u$, if there is no other state $t$ and coalition $K$ that is entitled to approve a change from $s$ to $t$, and each member of $K$ prefers $h(t)$ to $h(s)$. We denote the $\Gamma$-equilibria set at preference profile $u$ by $E(\Gamma, u)$.

Definition 2. An SCR $F$ is $\Gamma$-implementable if there exists a rights structure $\Gamma=(S, h, \gamma)$ such that for each $u \in \mathcal{P}, F(u)=h(E(\Gamma, u))$.

An SCR $F$ is implementable via the rights structure $\Gamma$ if at each preference profile $u$, the alternatives chosen by $F$ coincide with the outcomes of the $\Gamma$-equilibrium at $u$.

Comment: Many game-theoretic equilibrium concepts leave several components of the interaction unspecified. Similarly, in the definition of the $\Gamma$-equilibrium we leave out the following details of the interaction:

(1) From a current state $s$, more than one coalition may have the right to move. There is no priority ordering specified for these movements.

(2) If a coalition $K$ has the right to move, $K$ takes this move if and only if the movement is preferable. Issues such as preemptory moves-moving from a state $s$ to $t$ in order to avoid the movement of another coalition to a state $w$-are ruled out.

(3) An agent can be a member of several coalitions that have the right to move from one state to another. The procedure that agents follow to form a coalition is not specified. Similarly, for a given coalition, there might be more than one preferable movement. The collective decision rule to choose among these movements is also left unspecified.

(4) Coalitions are myopic in their movements, that is one state is eliminated in favor of another, which could also be eliminated by another coalition.

The benefits of leaving out such details are simplicity and wider applicability, unlike moreparticular designs. ${ }^{10}$ This is especially true for cooperative equilibrium notions. Another advantage of the design approach is that we are able to construct rights structures that are transparent and similar to institutional mechanisms, and in which some of the above details can be also

\footnotetext{
9 A state can represent a resource allocation, or an economic, political or legal arrangement. Furthermore, a state can itself be a constitution or legal code (Koray, 2000, Barbera and Jackson, 2004).

10 One can consult Chwe (1994) for a more detailed discussion.
} 
specified. Our analysis will shed light on which details are essential for implementing SCRs via simple rights structures, and which details can be omitted. To justify the following analysis, we will present several $\Gamma$-implementable rules and the rights structures that implement these rules.

\subsection{Examples}

In our first example we show that each Nash implementable SCR is also $\Gamma$-implementable. Note that, in this rather direct construction of the rights structure any complication present in the message space of the mechanism that implements the SCR, such as an integer game, is directly carried to the state space of the designed $\Gamma$. However, this should not mislead the reader, since in proving our Proposition 1, we show that, with a careful design, we can avoid this inheritance and design state spaces free of constructions similar to integer games. This later construction supports our claim that $\Gamma$-implementation provides an easily understandable framework for implementation. The construction in Example 1 aims to highlight the direct connection between Nash implementation and $\Gamma$-implementation.

Example 1 (Nash implementable SCRs). Let $F$ be an SCR that is Nash-implementable via a mechanism $(M, g)$. We show that $F$ is $\Gamma$-implementable. To see this let $\Gamma=(S, h, \gamma)$ be such that $S=M$ and $h=g$. Define $\gamma$ such that, for each distinct $s, t \in S$, if $s_{-i}=t_{-i}$ for some $i \in N$, then $\{i\} \in \gamma(s, t)$. If $s$ is different from $t$ by two or more components, then $\gamma(s, t)=\emptyset$. Now, it is easy to verify that $N E(M, g, u)=E(\Gamma, u)$.

A simple candidate for the state space of a rights structure is the set of alternatives, where the outcome function is the identity map. In this setting, a coalition $K \in \gamma(a, b)$ can be interpreted as follows: If the alternative $a$ is the current state, then $K$ can enforce the alternative $b$ as the new state. For the next two examples, the state space $S$ of the designed $\Gamma$ is the set of alternatives and the outcome function is the identity map. We show that the SCRs in Example 2 and Example 3, which fail to be Nash implementable, are $\Gamma$-implementable with this specific choice of the state space and the outcome function. In Example 4, we show that the Walrasian equilibrium outcome is also $\Gamma$-implementable in this restricted setup. Finally, in Example 5 we present a SCR that is $\Gamma$-implementable, and for which we have to choose a state space that is different than the alternative set. We revisit Example 5 in Section 6 while characterizing the SCRs that are implementable only by designing the code of rights. At this point, let us note that if one attempts to Nash implement an SCR via designing mechanisms, then constructions similar to integer games are often inevitable.

Example 2 (Pareto rule with veto power). Let $N=\{1,2,3\}$ and $A=\{a, b, c\}$. Given a preference profile $u$, for each alternative $x \neq a, x \in F(u)$ if and only if $x$ is Pareto efficient; $a \in F(u)$ if and only if $a$ is not only Pareto efficient, but also agent 1 prefers $a$ to $b$.

The rights structure $\Gamma$ depicted in Fig. 1 implements $F$. The code of rights is such that for each alternative $x$, the entire society $N$ has the right to move from $x$ to any other alternative $y$, and agent 1 has the right to move from $a$ to $b$. One can easily verify that $F$, which is not Nashimplementable, is implementable via this rights structure.

Example 3 (Choice with experts). Consider a decision maker who has a "fixed choice" $d$, and who also can choose among alternatives $a, b$, and $c$. Suppose he can consult consultants 1 and 


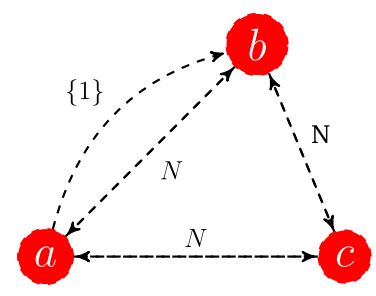

Fig. 1. $\Gamma$ for Pareto rule with veto power.

2 with expertise on alternatives $a$ and $b$, respectively. Now, given a "preference profile" $u$ of the experts, the decision maker wants to choose $a$, i.e. $a \in F(u)$ if and only if $a$ is top ranked by the first expert, $b \in F(u)$ if and only if $b$ is top ranked by the second expert, and $c \in F(u)$ if and only if $c$ is top ranked by both experts. To see that $F$ is $\Gamma$-implementable, consider the code of rights $\gamma$ where for each $x \in A$, we have $\gamma(a, x)=\{\{1\}\}, \gamma(b, x)=\{\{2\}\}$, and $\gamma(c, x)=\{\{1\},\{2\}\}$. One can easily verify that this rights structure implements $F$, which is not Nash-implementable (see Abreu and Sen, 1991).

Example 4 (Walrasian equilibrium). Consider any $2 \times 2$ pure exchange economy where agents have monotonic, continuous, and strictly convex preferences over the entire consumption space $R_{+}^{2}$. Given a strictly positive endowment vector $\omega$, let $F^{\omega}$ be the rule that chooses the Walrasian equilibrium allocations at each preference profile. Suppose each $a \in R_{+}^{2}$ is a state that corresponds to an allocation in the economy such that if $a$ is feasible $(a \leq \omega)$, then the first agent receives the bundle $a$ and the second agent receives the bundle $\omega-a$; if $a$ is not feasible, then the first agent receives $\omega$ and the second agent receives nothing. Next, consider the code of rights $\gamma$ such that for each $a, b \in R_{+}^{2}, \gamma(a, b)=\{\{1\},\{2\}\}$ if $a, b$, and $\omega$ are collinear or if $a$ is not feasible, and $\gamma(a, b)=\emptyset$ otherwise. One can easily verify that $F^{w}$ is implementable via this rights structure. $^{11}$

Example 5 (Tops rule). For each society $N$, alternative set $A$, and preference profile $u$, an alternative $x \in F(u)$ if and only if $x$ is top ranked by at least one of the agents. To see that $F$ is $\Gamma$-implementable, let $S$ be the set of all alternative-agent pairs. We can interpret a state $(x, i)$ as a claim for $x$ is top ranked by $i$. For each $(x, i) \in S$, let $h(x, i)=x$. Let code of rights $\gamma$ be such that for each $(x, i),(y, j) \in S \times S$, if $i \neq j$ then $\gamma((x, i),(y, j))=\emptyset$, and if $i=j$ then $\gamma((x, i),(y, j))=\{\{i\}\}$. To see that the suggested rights structure implements $F$, let $x$ be an alternative top ranked by agent $i$ at the given preference profile. Since no agent other than $i$ can move from $(x, i)$, it is an equilibrium state. Moreover, if an alternative $y$ is not top ranked, then from each state $(y, j)$, agent $j$ prefers to move a state where the associated outcome is his top ranked alternative. Hence, no state with outcome $y$ can be an equilibrium state.

\footnotetext{
11 Here, the code of rights enable agents to eliminate an allocation by moving to another one that can be infeasible. It may be more natural to allow agent to move from an allocation only to a feasible one. If we define the code of rights as to allow movements only among feasible allocations $a$ and $b$ that are collinear with $\omega$, then we obtain a slightly larger set than the Walrasian allocations as the equilibria. Hurwicz et al. (1995) calls this set the constrained Walrasian allocations, and show that these allocations are Nash implementable although Walrasian allocations are not. This larger set coincides with the Walrasian allocations if each agent's demand for a good is constrained to be no greater than the total endowment of the good.
} 


\section{Monotonicity and $\Gamma$-implementation}

In classical implementation via mechanisms, Maskin (1985) shows that every Nashimplementable SCR is monotonic, and monotonicity combined with no veto power condition is sufficient for Nash implementability in the presence of at least three agents. To explain monotonicity, suppose that an alternative $a$ is chosen at a preference profile $u^{1}$ according to a given SCR $F$. Now, if $a$ does not fall in anyone's ranking relative to any other alternative in going from profile $u^{1}$ to profile $u^{2}$, then monotonicity requires that $a$ also be chosen at $u^{2}$. As for a full characterization of Nash implementability in the presence of at least three agents, Danilov (1992) proposes the notion of essential monotonicity, which strengthens monotonicity by requiring that once $a$ is chosen, then $a$ continues to be chosen even if some non-essential alternatives are moved on top of $a .{ }^{12}$ Danilov shows that essential monotonicity is both necessary and sufficient for Nash implementability in the presence of at least three agents.

Here, we introduce the image monotonicity condition, which is slightly stronger than Maskin's monotonicity, but weaker than Danilov's essential monotonicity. For a given SCR $F$, an alternative $x$ is in the image of $F$ if there exists a preference profile at which $F$ chooses $x$. Now, for each alternative $a$, if $a$ is chosen at a preference profile $u^{1}$, and in going from profile $u^{1}$ to profile $u^{2}, a$ does not fall in anyone's ranking relative to any other alternative that is in the image of $F$, then image monotonicity requires that $a$ is also chosen at $u^{2}$. Intuitively, one can consider an alternative as "dummy" if there is no preference profile at which that alternative is chosen. Image monotonicity strengthens monotonicity by requiring that once $a$ is chosen, then $a$ continues to be chosen even if some dummy alternatives, which can never be chosen, are moved on top of $a$. In relation to Danilov's essential monotonicity, our essential alternatives are the ones that are not dummies.

We show that an SCR $F$ is $\Gamma$-implementable if and only if $F$ is image monotonic. It follows from this result that a unanimous SCR is $\Gamma$-implementable if and only if $F$ is Maskin monotonic. ${ }^{13}$ Moreover, it follows from the proof of this proposition that any $\Gamma$-implementable SCR can be implemented via an individual based (IB) rights structure, which requires for each distinct state $s, t$, if $\gamma(s, t)$ is nonempty, then $\gamma(s, t)$ consists of singleton coalitions. This rules out issue (3), and partially issue (1), both of which we listed as part of our discussion in Section 2.

Before proceeding to the formal definition of image monotonicity, let us introduce some useful notation. Let $I(F)$ denote the image of $F$, i.e. $I(F)=\{a \in A: a \in F(u)$ for some $u \in \mathcal{P}\}$. The lower contour set of $u_{i}$ with respect to $a \in A$, denoted by $\mathrm{L}\left(u_{i}, a\right)$, is the set of alternatives to which $a$ is preferred by agent $i$, i.e. $\mathrm{L}\left(u_{i}, a\right)=\left\{b \in A: a u_{i} b\right\}$. An SCR $F$ is Maskin monotonic if for each $u^{1}, u^{2} \in \mathcal{P}$ and $a \in F\left(u^{1}\right)$, we have $a \in F\left(u^{2}\right)$ whenever for every $i \in N, \mathrm{~L}\left(u_{i}^{1}, a\right) \subseteq$ $\mathrm{L}\left(u_{i}^{2}, a\right)$.

Image monotonicity: An SCR $F$ is image monotonic, if for each $u^{1}, u^{2} \in \mathcal{P}$, and each $a \in$ $F\left(u^{1}\right)$, we have $a \in F\left(u^{2}\right)$ whenever for every $i \in N$,

$$
\mathrm{L}\left(u_{i}^{1}, a\right) \cap I(F) \subseteq \mathrm{L}\left(u_{i}^{2}, a\right)
$$

\footnotetext{
12 Formally, an alternative $x$ is essential at a preference profile $u$ for agent $i$, denoted by $x \in \operatorname{Ess}(i, u)$, if $x$ is chosen whenever $x$ is moved to the top in the rankings of all other agents but is kept as it is in agent $i$ 's ranking. Now, for each alternative $a$, if $a$ is chosen at a preference profile $u^{1}$, and in going from profile $u^{1}$ to profile $u^{2}$ for each agent $i$, $a$ does not fall in $i$ 's ranking relative to any other alternative that is in $\operatorname{Ess}\left(i, u^{1}\right)$, then essential monotonicity requires that $a$ also be chosen at $u^{2}$.

13 One notable aspect of this characterization is its simplicity and validity even with only two agents. This is in contrast to the characterizations of Nash implementability.
} 
Proposition 1. Given an SCR F, the following are equivalent:

\author{
i. $F$ is image monotonic \\ ii. $F$ is $\Gamma$-implementable \\ iii. $F$ is $\Gamma$-implementable via an individual based rights structure.
}

Corollary 1. A unanimous SCR F is $\Gamma$-implementable if and only if $F$ is Maskin monotonic.

To prove Proposition 1 as well as several other results to follow, we use the canonical rights structure $\Gamma^{F}=\left(S^{F}, h^{F}, \gamma^{F}\right)$ designed as follows. Let $F$ be a given SCR, the state space $S^{F}$ is defined as the graph of $F$, i.e. $S^{F}=\{(a, u) \in A \times \mathcal{P}: a \in F(u)\}$. The outcome function $h^{F}$ maps each $(a, u) \in S^{F}$ to $a$. The code of rights $\gamma^{F}$ will entitle an agent $i$ to move from state $(a, u)$ to another state $(b, v)$ if and only if $i$ prefers $a$ to $b$ at $u$, i.e. $a u_{i} b$.

From the view of the designer, the state space of a canonical rights structure is a natural choice, in that each state carries a candidate for being the true preference profile, which is unknown by the designer. In this vein, we can interpret a state $(a, u)$ as a proposal for alternative $a$ with supporting proposition $u$. This supporting proposition can be thought of as an argument: "Alternative $a$ should be chosen, since the true preference profile is $u$." Given this interpretation of a state, agents who can credibly refute the supporting argument should have the right to move from state $(a, u)$ to another state $(b, v)$ in which alternative $b$ is chosen. Note that these are the agents who prefer $a$ to $b$ at $u$, in that if an agent $i$ prefers $a$ to $b$ at $u$ and the statement was true $\left(u_{i}\right.$ was the true preference of $i)$, then he has no incentive to move from $(a, u)$ to $(b, v)$. In line with this interpretation, the canonical code of rights entitles an agent $i$ to move from a state $(a, u)$ to another state $(b, v)$ if and only if $i$ prefers $a$ to $b$ at $u{ }^{14}$ As for another interpretation of a state $(a, u)$, the preference profile $u$ can represent the "conflict of interest" relationships in the sense that for each agent $i$ and pair of alternatives $a$ and $b$, if $a u_{i} b$, then this means agent $i$ has some vested interest to move from outcome $a$ to outcome $b$. Then, according to the canonical code of rights an agent is entitled to move from a state $(a, u)$ to another state $(b, v)$ only if there is no conflict of interest. ${ }^{15}$

At a formal level, the canonical rights structure is similar to a Maskin type of mechanism without an integer game. To see the connection, recall that the strategy space of each agent in a Maskin type mechanism consists of alternative, preference profile, and integer triplets $(a, u, n)$. The outcome function is designed such that if every player but agent $i$ plays the same triplet $(a, u, n)$, and $i$ plays $(b, v, m)$, then the outcome is $b$ if and only if $a$ is better than $b$ at $u_{i}$. In the language of rights structure this corresponds to: $i$ is entitled to move from state $(a, u)$ to another state $(b, v)$ if and only if $a u_{i} b$.

In the following example, we tailor the canonical rights structure to implement the majority rule. We prove Proposition 1 by using canonical structures and following a line of simple reasoning as used in this example.

\footnotetext{
14 Having a supporting argument as a part of a state is similar to the notion of evidence recently used in implementation (Kartik and Tercieux, 2012, Bull and Watson, 2007). The basic difference between our notion and theirs is that although evidence structures are specified as contingent on the preference profiles, a state comes with the same supporting argument independent of the true preferences. In Section 7, we introduce the strategic counterpart of $\Gamma$-implementability, and then compare and contrast the related studies.

15 This is similar to excluding a candidate's spouse from his promotion committee or long term collaborators from reviewing each other's articles for publication.
} 


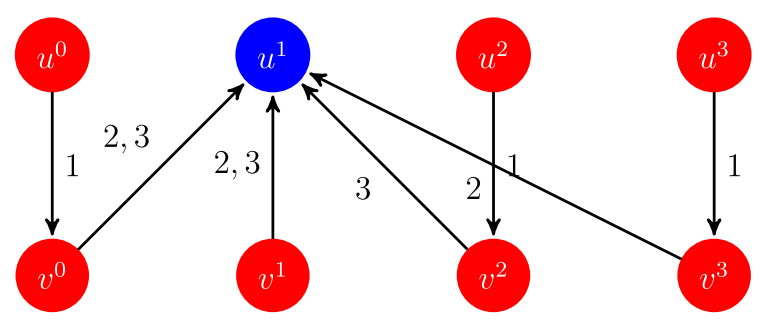

Fig. 2. Majority rule.

Example 6 (Majority rule). Let $N=\{1,2,3\}$ and $A=\{a, b\}$. For each preference profile $u$, $F(u)=a$ if and only if $a$ is preferred to $b$ by at least two agents. Notice that there are four profiles $\left\{u^{0}, u^{3}, u^{2}, u^{1}\right\}$ where $a$ is chosen by $F$, since $a$ is preferred to $b$ by $N,\{1,2\},\{1,3\}$, and $\{2,3\}$, respectively. Similarly, let $\left\{v^{0}, v^{3}, v^{2}, v^{1}\right\}$ be the profiles where the roles of $a$ and $b$ are changed, so that $b$ is chosen by $F$. Now, consider the canonical rights structure $\Gamma^{S}=\left(S^{F}, h^{F}, \gamma^{F}\right)$ tailored for this rule. Namely, we have $S^{F}=\left\{\left(a, u^{m}\right),\left(b, v^{m}\right)\right\}_{m=0}^{3}$, where the outcome function $h^{F}$ maps each $\left(a, u^{m}\right)$ to $a$ and each $\left(b, v^{m}\right)$ to $b$. Now, the code of rights $\gamma^{c}$ entitles any agent $\{i\}$ to move from any state $\left(a, u^{m}\right)$ to another state $\left(b, v^{r}\right)$ if and only if $a u_{i}^{m} b$. To illustrate how $\Gamma^{F}$ implements $F$, as depicted in Fig. 2, suppose the true preference profile is $u^{1}$, so we have $F\left(u^{1}\right)=a$. Now, consider the state $\left(a, u^{1}\right)$. Only agent 1 prefers to move from $\left(a, u^{1}\right)$ to a state with outcome $b$, but by the design of $\gamma^{F}$, agent 1 does not have the right to move. Hence, $\left(a, u^{m}\right) \in E\left(\Gamma^{F}, u^{m}\right)$. On the other hand, for each $v^{r}$, since at least two agents prefer $b$ to $a$ at $v^{r}$, there necessarily exists $i \in\{2,3\}$ such that $b v_{i}^{r} a$ and $a u_{i}^{1} b$. By the design of $\gamma^{F}$ this means $i$ is entitled to move from $\left(b, v^{r}\right)$ to $\left(a, u^{m}\right)$. Since $a u_{i}^{1} b$, it follows that $\left(b, v^{r}\right) \notin E\left(\Gamma^{F}, u^{1}\right)$. Thus, we obtain $h^{F}\left(E\left(\Gamma^{F}, u^{1}\right)\right)=a$.

Proof of Proposition 1. $(i \Rightarrow$ iii) Let $F$ be an image-monotonic social choice rule. Let $\Gamma$ be the canonical rights structure that is IB. Formally, for each $((a, u),(b, v)) \in S^{F} \times S^{F}$ and $i \in N$ we have,

$\{i\} \in \gamma^{F}((a, u),(b, v))$ if and only if $a u_{i} b$.

We will show that for each $u^{2} \in \mathcal{P}$ and $a \in A$, we have $a \in F\left(u^{2}\right)$ if and only if there exists $\left(a, u^{1}\right) \in E\left(\Gamma^{F}, u^{2}\right)$. Given $u^{2} \in \mathcal{P}$, suppose $a \in F\left(u^{2}\right)$. We will show that $\left(a, u^{2}\right) \in$ $E\left(\Gamma^{F}, u^{2}\right)$. Now, for each $(b, v) \in S^{F}$ and agent $\{i\} \in \gamma^{F}\left(\left(a, u^{2}\right),(b, v)\right)$, by the design of $\gamma^{c}$, we have $a u_{i}^{2} b$. Thus, we obtain $\left(a, u^{2}\right) \in E\left(\Gamma^{F}, u^{2}\right)$. Conversely, suppose there exists $\left(a, u^{1}\right) \in$ $E\left(\Gamma^{F}, u^{2}\right)$. Note that for each $b \in I(F)$, there exists a preference profile $v$ such that $(b, v) \in S^{F}$. Since we have $\left(a, u^{1}\right) \in E\left(\Gamma^{F}, u^{2}\right)$, this means that for each $i \in N$ and $b \in I(F)$, if $a u_{i}^{1} b$ then $a u_{i}^{2} b$. That is, $\mathrm{L}\left(u_{i}^{1}, a\right) \cap I(F) \subseteq \mathrm{L}\left(u_{i}^{2}, a\right)$. Since $a \in F\left(u^{1}\right)$, it follows from image monotonicity that $a \in F\left(u^{2}\right)$.

(iii $\Rightarrow$ ii) Follows directly.

$(i i \Rightarrow i)$ Let $F$ be a $\Gamma$-implementable social choice rule, and let $\Gamma=(S, h, \gamma)$ be a rights structure that implements $F$. Let $u^{1}, u^{2} \in \mathcal{P}$ be such that $a \in F\left(u^{1}\right)$, and suppose for every $i \in N$, we have $\mathrm{L}\left(u_{i}^{1}, a\right) \cap I(F) \subseteq \mathrm{L}\left(u_{i}^{2}, a\right)$. Now, we will show that $a \in F\left(u^{2}\right)$. Suppose $a \notin$ $F\left(u^{2}\right)$. Since $\Gamma$ implements $F$ and $a \in F\left(u^{1}\right)$, there is some $s \in E\left(\Gamma, u^{1}\right)$ with $h(s)=a$. Since $a \notin F\left(u^{2}\right), s \notin E\left(\Gamma, u^{2}\right)$. It follows that there exist $t \in S$ and a coalition $K$ such that $h(t)=b$ for some $b \neq a$, where $K \in \gamma(s, t)$ and $K$ prefers $b$ to $a$ at $u^{2}$. First, we claim that $b \in I(F)$. 


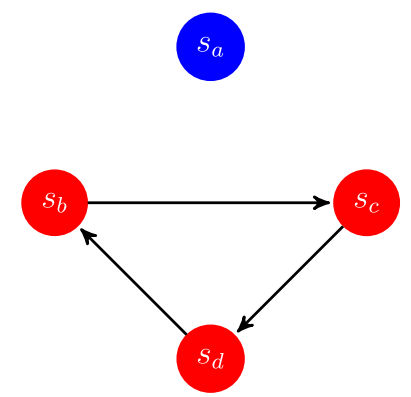

Fig. 3. $\Gamma$ with a cycle of nonequilibrium states.

To see this, consider a preference profile $u^{b}$ where every agent top ranks $b$. Since $t \in S$ with $h(t)=b$, there is no agent that prefers a change of state from $t$ to another state. Thus, $t \in$ $E\left(\Gamma, u^{b}\right)$. Since $F\left(u^{b}\right)=E\left(\Gamma, u^{b}\right)$, we obtain $b \in F\left(u^{b}\right)$. Now, since for every $i \in K, \mathrm{~L}\left(u_{i}^{1}, a\right) \cap$ $I(F) \subseteq \mathrm{L}\left(u_{i}^{2}, a\right)$ and $b u_{i}^{2} a$, we obtain that $K$ prefers $b$ to $a$ at $u^{1}$. Since $K \in \gamma(s, t)$, this contradicts $s \in E\left(\Gamma, u^{1}\right)$.

\subsection{Convergence to equilibrium}

Most game-theoretic equilibrium concepts leave the convergence dynamics from a nonequilibrium strategy to an equilibrium strategy unspecified. However, one can require the designed constructs - such as the game forms or the rights structures - to be endowed with well-defined convergence properties. Although for many equilibrium concepts it is the "beliefs" of the agents that direct them to play equilibrium strategies, for $\Gamma$-equilibrium it is the "allowed" and "desired" movements among states that carry the agents to the equilibrium. Therefore, questions related to the possibility of convergence from nonequilibrium states to equilibrium states is particularly important in our design framework. In this section, we show that every unanimous SCR that is $\Gamma$-implementable is implementable such that each nonequilibrium state is connected to each equilibrium state via a path of movements among states. Moreover, we observe that the length of this path can be guaranteed to be at most two.

Let $F$ be an SCR and $\Gamma$ a rights structure that implements $F$. In general, when starting from any nonequilibrium state it may not be possible to reach an equilibrium state. Put differently, as depicted in Fig. 3, the only possible movement from a nonequilibrium state might be directly into a cycle consisting of nonequilibrium states. In such a case, one can argue that the equilibrium state would prevail only if the society has the equilibrium state as the current state. To avoid such a restrictive conclusion, one can require that the following robustness condition be satisfied by a rights structure.

Path Existence: For each preference profile $u$, each state $s_{0} \notin E(\Gamma, u)$, and each state $s \in$ $E(\Gamma, u)$, there exists a path (a finite sequence of states) $s_{0}, \ldots, s_{m}$ with $s_{m}=s$ such that for each $k \in\{1, \ldots, m\}$, there is a coalition $K \in \gamma\left(s_{k-1}, s_{k}\right)$ with $h\left(s_{k}\right) u_{K} h\left(s_{k-1}\right)$.

In implementing a SCR via a rights structure, an important detail that is left unspecified is how is the initial state determined. If the rights structure in use satisfies the path existence requirement, then each equilibrium state can be reached from any nonequilibrium state via a sequence of states such that the passage from each state in the sequence to the next one is justified both rights- and preference-wise. Thus, the given SCR is implemented regardless of which state happens to be the initial state. 
Corollary 2. Let $F$ be a $\Gamma$-implementable SCR. If $F$ is unanimous, then $F$ is implementable via a rights structure that satisfies path existence such that the length of each path is at most two.

Proof. Let $F$ be a unanimous and $\Gamma$-implementable SCR. We show that the canonical rights structure $\Gamma^{F}$ that implements $F$ also satisfies the path existence condition such that the length of each path is at most two. To see this, consider a preference profile $u$. Now, for each state $\left(a, u^{\prime}\right)$, if $\left(a, u^{\prime}\right) \notin E(\Gamma, u)$, then, it follows from the definition of $\Gamma^{F}$ that there exist $b \in A$ and $i \in N$ such that $a u_{i}^{\prime} b$ and $b u_{i} a$. If $b \in F(u)$, then we obtain the desired path of length one between $\left(a, u^{\prime}\right)$ and $(b, u)$. If not, let $u^{b}$ be a preference profile at which $b$ is top ranked by each agent. Since $F$ is unanimous, $F\left(u^{b}\right)=b$. Moreover, we know that agent $i$ has the right to move from $\left(a, u^{\prime}\right)$ to $\left(b, u^{b}\right)$ and $b u_{i} a$. Thus, the movement from $\left(a, u^{\prime}\right)$ to $\left(b, u^{b}\right)$ constitutes the first part of our desired path. Next to define the second part, pick any $\left(x, u^{\prime \prime}\right) \in E(\Gamma, u)$. By our choice of $u^{b}$, each agent has the right to move from $\left(b, u^{b}\right)$ to $\left(x, u^{\prime \prime}\right)$. Now, we have to show that at least one agent prefers this movement. Since $\Gamma^{F}$ implements $F$ and $\left(x, u^{\prime \prime}\right) \in E(\Gamma, u), x \in F(u)$. Since $F$ is image monotonic and unanimous, $F$ is also Pareto efficient. Since $x \in F(u)$, it follows that there exists $j \in N$ with $x u_{j} b$. Thus, we obtain the desired path of length two, which connects $\left(a, u^{\prime}\right)$ to $\left(x, u^{\prime \prime}\right)$ by passing through $\left(b, u^{b}\right)$.

Implementability via a rights structure that satisfies path existence can be thought of as the counterpart of recurrent implementation proposed by Cabrales and Serrano (2011) ${ }^{16}$ in which agents are assumed to myopically adjust their actions by randomly starting from an arbitrary joint strategy profile in the direction of better-responses. In this setting, an SCR is said to be implementable if for each preference profile, all the outcomes of the SCR coincide with the limiting outcomes of the better-response dynamics of a mechanism. Cabrales and Serrano show that monotonicity, together with the "no worst alternative property" 17 is key for characterizing the implementable SCRs in the presence of at least three agents. We show that in our framework monotonicity, together with unanimity is sufficient for a similar type of implementability.

\subsection{Externally stable $\Gamma$-implementation}

Most game-theoretic equilibrium concepts are myopic in the following sense: A joint strategy is eliminated from the equilibria whenever a player prefers a unilateral deviation; similarly, a joint strategy is an equilibrium whenever there is no preferable unilateral deviation. However, one can question the myopia of an agent eliminating one strategy in favor of another that could also be eliminated. Several game theoretic studies address this question. As an incomplete list we can count social decision systems by Rubinstein (1980), social situations by Greenberg (1990), largest consistent set by Chwe (1994), dynamically stable sets by Acemoglu et al. (2012), and the earlier stability discussions of von Neumann and Morgenstern (1953), Aumann and Maschler (1964), but none of these studies lie in a design framework.

Our $\Gamma$-equilibrium notion can also be criticized as being myopic, in that agents use their rights to approve a change from a state to another whenever the latter outcome is better, regardless of whether it is an equilibrium state or not. Departing from this point, we make deviations stringent, thus making the stability notion looser. In particular, to block a nonequilibrium state with an

\footnotetext{
16 We are grateful to an anonymous referee for bringing this study to our attention.

17 This property requires that for each preference profile, there is a worse alternative for each agent than each alternative prescribed by the SCR.
} 
undesirable outcome, we require the existence of an equilibrium state to which an agent both has the right and the incentive to move.

Externally Stable $\Gamma$-implementation: An SCR $F$ is said to be implemented via an externally stable rights structure $\Gamma=(S, h, \gamma)$, if

i. $\Gamma$ implements $F$, and

ii. for each $u \in \mathcal{P}$, and $s \notin E(\Gamma, u)$ with $h(s) \notin F(u)$, there exist $t \in E(\Gamma, u)$ and $i \in N$ such that $\{i\} \in \gamma(s, t)$ and $h(t) u_{i} h(s)$.

In the course of implementation via mechanisms, the appended integer game makes sustaining external stability seem implausible. However, our framework makes it tractable to design rights structures which are externally stable. These right structures allow the designer to implement desirable outcomes even with agents who can be farsighted. In the following analysis, we provide a characterization of SCRs that are farsightedly implemented via externally stable rights structures. The following strengthening of image monotonicity—which carries over the well-known independence of irrelevant alternatives condition (Arrow, 1967) - into the realm of monotonicity, characterizes this class of SCRs. Winner monotonicity strengthens monotonicity by requiring that once $a$ is chosen, $a$ continues to be chosen even if the alternatives not chosen at the new preference profile are moved on top of $a$. Put differently, if $a$ is chosen at a preference profile, then $a$ continues to be chosen in any other preference profile if the chosen alternatives at the new profile that were initially ranked below $a$ are still ranked below $a$ at the new profile. Although winner monotonicity is a rather demanding monotonicity condition, in our examples, Pareto rule, tops rule, and majority rule satisfy winner monotonicity. One can easily verify that our Examples 2 and 3 in Section 3.1 fail to satisfy winner monotonicity.

Winner Monotonicity: An SCR $F$ is winner monotonic if for each $u^{1}, u^{2} \in \mathcal{P}$ and $a \in F\left(u^{1}\right)$, we have $a \in F\left(u^{2}\right)$ whenever for every $i \in N$

$$
\mathrm{L}\left(u_{i}^{1}, a\right) \cap F\left(u^{2}\right) \subseteq \mathrm{L}\left(u_{i}^{2}, a\right)
$$

Proposition 2. An SCR F is implemented via an externally stable rights structure if and only if $F$ is winner monotonic.

Proof. $(\Rightarrow)$ Let SCR $F$ be implemented via an externally stable rights structure $\Gamma$. Let $u^{1}, u^{2} \in \mathcal{P}$ such that $a \in F\left(u^{1}\right)$. Since $a \in F\left(u^{1}\right)$, there exists $s \in E\left(\Gamma, u^{1}\right)$ such that $h(s)=a$. Suppose for every $i \in N$ we have $\mathrm{L}\left(u_{i}^{1}, a\right) \cap F\left(u^{2}\right) \subseteq \mathrm{L}\left(u_{i}^{2}, a\right)$. It follows that for every $i \in N$ and $b \in F\left(u^{2}\right)$, if $a u_{i}^{1} b$ then $a u_{i}^{2} b$ as well. Now, since $s \in E\left(\Gamma, u^{1}\right)$, for each $b \in F\left(u^{2}\right)$ and $t \in S$ with $h(t)=b$ there is no agent $\{i\} \in \gamma(s, t)$ with $h(t) u_{i} h(s)$. It follows from external stability that we have $s \in E\left(\Gamma, u^{2}\right)$, hence $a \in F\left(u^{2}\right)$.

$(\Leftarrow)$ Let $F$ be winner monotonic. Let $\Gamma=(S, h, \gamma)$ be the canonical rights structure. From Proposition 1, we know that $\Gamma$ implements $F$. Now we will show that $\Gamma$ is externally stable. Let $u^{1} \in \mathcal{P}$ and $a \notin F\left(u^{1}\right)$. If $a \notin I(F)$, then there is no state $s \in S$ with $h(s)=a$, so we are done. Suppose $a \in I(F)$ and let $(a, v) \in S$. We claim that, there exist $i \in N$ and $b \in F\left(u^{1}\right)$ such that $b u_{i}^{1} a$ and $a v_{i} b$. If not, for each $i \in N$, we have $\mathrm{L}\left(v_{i}, a\right) \cap F\left(u^{1}\right) \subseteq \mathrm{L}\left(u_{i}^{1}, a\right)$. It follows from winner monotonicity that $a \in F\left(u^{1}\right)$, which is a contradiction. Now for such $i \in N$ and $b \in F\left(u^{1}\right)$, by the design of $\gamma$, we have $\{i\} \in \gamma\left((a, v),\left(b, u^{1}\right)\right)$. Thus the conclusion follows. 


\section{Rights structures underlying implementability via mechanisms}

In this section we aim to identify the rights structures underlying classical implementation via mechanisms. If an SCR is Nash or strong Nash-implementable, then it is $\Gamma$-implementable as well. Thus we focus on these SCRs to determine the further properties that the rights structures implementing them possess. These results shed light on when is it possible to add more details to rights structures in order to present the agents' interaction in game form.

\subsection{Nash implementation}

First we introduce a transitivity condition that pertains to rights structures. Then, we show that in the presence of at least three agents, the class of Nash-implementable SCRs coincides with the class of SCRs that can be implemented via individual based and individually transitive right structures. We have defined individual based rights structure at the beginning of Section 4 . Individual transitivity requires that if an individual has a right to move from a state $s$ to another state $t$ and to move from state $t$ to a third state $w$, then the same individual should have the right to move from state $t$ to state $w$ directly. Individual transitivity eliminates incentives for preemptive movements at the individual level, since any state that can be obtained via a chain of movements among states can be directly obtained. Next, we present the formal definition of individual transitivity and the result.

(IT) A rights structure $\Gamma=(S, h, \gamma)$ is individually transitive if for each distinct $s, t, w \in S$ and $i \in N$, if $\{i\} \in \gamma(s, t) \cap \gamma(t, w)$, then $\{i\} \in \gamma(s, w)$.

Proposition 3. In the presence of at least three agents, an SCR F is Nash-implementable if and only if $F$ is implementable via an individual based and individually transitive rights structure.

Proof. $(\Rightarrow)$ It follows from Example 1 that if an SCR is Nash implementable, then it is also $\Gamma$-implementable. Since the $\Gamma$ constructed in Example 1 is clearly IB and IT, this part of the proposition follows.

$(\Leftarrow)$ Suppose there is an IB and IT rights structure $\Gamma=(S, h, \gamma)$ that implements $F$. If $S$ consists of only two states $s, s^{\prime}$, then let us consider another rights structure with the only difference being an additional state $s^{\prime \prime}$, which is a clone of $s$ for the outcome function and the code of rights. Notice that this new rights structure, with three states, would implement $F$ as well. Hence, we can w.l.o.g. assume that $S$ contains at least three different states. Next, we define the mechanism to implement $F$ under Nash equilibrium. For each $i \in N$, let $M_{i}=S \times N \times \mathbb{Z}_{+}$, where $N=\{1, \ldots, n\}$ stands for the set of agents ordered accordingly, and $\mathbb{Z}_{+}$stands for the set of positive integers. That is, each agent announces a state, anagent, and a positive integer. Let the outcome function $g$ be such that:

(1) If everybody announces the same state $s$, then we have $g(m)=h(s)$.

(2) Suppose everybody but $i$ announces the state $s$, and $i$ announces the state $s^{\prime}$. If $\{i\} \in \gamma\left(s, s^{\prime}\right)$ then $g(m)=h\left(s^{\prime}\right)$; if not then $g(m)=h(s)$.

(3) Otherwise, let $i$ be the agent with the minimum index among the ones who announce the highest integer. Let $j$ be the agent pointed at by agent $i$. Let $s^{\prime \prime}$ be the state announced by agent $j$. Therefore we have $g(m)=h\left(s^{\prime \prime}\right)$. 
Now, consider the mechanism $(M, g)$. For each $u \in \mathcal{P}$ and $s \in E(\Gamma, u)$, consider any strategy profile $m$ such that for every $i \in N, m_{i, 1}=s$. Since $s \in E(\Gamma, u)$, we have for every $s^{\prime} \neq s$ and $i \in N$, if $h\left(s^{\prime}\right) u_{i} h(s)$ then $\{i\} \notin \gamma\left(s, s^{\prime}\right)$. It follows that $m \in N E(M, g, u)$.

Next we show that for each $m \in N E(M, g, u)$, we have $g(m) \in h(E(\Gamma, u))$. By contradiction, suppose there exists $m \in M$ such that $g(m) \notin h(E(\Gamma, u))$. It follows that for each $s \in S$ with $g(m)=h(s)$, we have $s \notin E(\Gamma, u)$. Combined with IB, it follows that there exist $s^{\prime} \in S$ and $i \in N$ such that $\{i\} \in \gamma\left(s, s^{\prime}\right)$ and $h\left(s^{\prime}\right) u_{i} h(s)$. We show the contradiction by showing that agent $i$ can deviate to obtain $h\left(s^{\prime}\right)$.

Case 1: Suppose $m$ is as in (1). Then let agent $i$ announce $s^{\prime}$ instead of $s$. Since $\{i\} \in \gamma\left(s, s^{\prime}\right)$, the outcome is $h\left(s^{\prime}\right)$.

Case 2: Suppose $m$ is as in (2). Let $t$ stand for the state announced by everyone but a single agent who announces another state $w$. Now there are two possibilities. First, suppose that $w=s$ and there is an agent $\{j\} \in \gamma(t, s)$. Suppose $m$ is such that agent $i$ announces the state $t$. Now agent $i$ can deviate to obtain $h\left(s^{\prime}\right)$ as follows: If $t \neq s^{\prime}$, then agent $i$ announces the state $s^{\prime}$, the highest integer, and points at himself. If $t=s^{\prime}$, since there are at least three states, agent $i$ announces a third state and the highest integer. Since $n \geq 3$, there is an agent who announces $s^{\prime}$ and agent $i$ points at one of those agents. Next, suppose that $t=s$ and $\gamma(s, w)=\emptyset$. Now, agent $i$ can deviate to obtain $h\left(s^{\prime}\right)$ as follows: If $w \neq s^{\prime}$, then let agent $i$ announce state $s^{\prime}$, the highest integer, and point at himself. Since $\{i\} \in \gamma\left(s, s^{\prime}\right)$, the new outcome is $h\left(s^{\prime}\right)$. If $w=s^{\prime}$, then let agent $i$ announce a third state, the highest integer, and point at the agent who announces $s^{\prime}$.

Case 3: Suppose $m$ is as in (3). Now, agent $i$ can deviate to obtain $h\left(s^{\prime}\right)$ as follows: If no one has announced $s^{\prime}$ then agent $i$ announces the state $s^{\prime}$, the highest integer, and points at himself. If some agent $j$ has announced $s^{\prime}$, then agent $i$ announces the highest integer and points at $j$. Thus, we reach the contradiction that $m \notin N E(M, g, u)$.

We know from Proposition 1 that each $\Gamma$-implementable SCR is implementable via an individual based code of rights. This observation, together with Proposition 3, indicates that what distinguishes Nash-implementable SCRs from the rest is individually transitivity of the underlying rights structure being individually transitive. Now, let us turn back to our examples in Section 3.1 that are shown to be $\Gamma$-implementable. When considering these examples, the reader can verify that our assumptions in Proposition 3 are necessary for the result and independent from each other. The SCR in our Example 2 is not Nash implementable, since the designed $\Gamma$ is IT but not IB. Although the $\Gamma$ designed to implement the SCR in Example 3 is both IB and IT, the SCR is not Nash implementable, in that there are only two agents in the SCR. Since the $\Gamma$ designed to implement the tops rule in our Example 5 is IB and IT, it follows from Proposition 3 that it is Nash implementable in the presence of at least three agents.

\subsection{Strong Nash implementation}

First, let us introduce the definitions related to strong Nash implementability. Given a mechanism $(M, g)$, for each coalition $K$, let $M_{K}=\Pi_{i \in K} M_{i}$. For each preference profile $u$ and $m, m^{\prime} \in M$, we write $g(m) u_{K} g\left(m^{\prime}\right)$ if for each $i \in K, g(m) u_{i} g\left(m^{\prime}\right)$. A strategy profile $m$ is a strong Nash equilibrium of the game $(M, g, u)$ if for each coalition $K$ and $m_{K}^{\prime} \in M_{K}$, there exists $i \in K$ such that $g(m) u_{i} g\left(m_{K}^{\prime}, m_{-K}\right)$. The set of strong Nash equilibrium in the game $(M, g, u)$ is denoted by $\operatorname{SNE}(M, g, u)$. An SCR $F$ is implementable in strong Nash equilibrium if there is a mechanism $(M, g)$ such that for each preference profile $u$, we have $F(u)=g(\operatorname{SNE}(M, g, u))$. 
Here, we first propose two properties pertaining to rights structures. The first one, being coalitionally ascending, requires that if an agent has a right to move from one state to another, then whenever this agent belongs to a coalition, that coalition should have the same right as well. The second property, coalitional transitivity, is the counterpart of individual transitivity, and requires that if a coalition has a right to move from a state $s$ to another state $t$ and to move from state $t$ to a third state $w$, then this coalition should have the right to move from state $t$ to state $w$ directly. Next, we present the formal definitions and the result.

(CA) A rights structure $\Gamma=(S, h, \gamma)$ is coalitionally ascending if for each coalition $K$, $i \in K$, and distinct $s, t \in S$,

$$
\text { if }\{i\} \in \gamma(s, t) \text {, then } K \in \gamma(s, t)
$$

(CT) A rights structure $\Gamma=(S, h, \gamma)$ is coalitionally transitive if for each coalition $K, i \in K$, and distinct $s, t, w \in S$,

$$
\text { if } K \in \gamma(s, t) \text { and } K \in \gamma(t, w) \text {, then } K \in \gamma(s, w)
$$

Proposition 4. In the presence of at least three agents, an SCR F is strong Nash implementable if and only if $F$ is implementable via a coalitionally ascending and coalitionally transitive rights structure.

Proof. $(\Rightarrow)$ Let $F$ be an SCR that is strong Nash implementable via a mechanism $(M, g)$. Let $\Gamma=(S, h, \gamma)$ be such that $S=M$ and $h=g$. To define $\gamma$, for each $s, t \in S$ let $K=\{i \in N$ : $\left.s_{-i} \neq t_{-i}\right\}$. Now define the code of rights $\gamma$ such that only the coalition $K \in \gamma(s, t)$. By this construction, $\Gamma$ is CA, CT and implements $F$.

$(\Leftarrow)$ Suppose there is a CA and CT rights structure $\Gamma=(S, h, \gamma)$ that implements $F$. If $S$ consists of only two states $s, s^{\prime}$, then let us consider another rights structure with an additional state $s^{\prime \prime}$ that is a clone of $s$ for the outcome function and the code of rights.

Next, we define a mechanism for implementing $F$. For each $i \in N$, let $M_{i}=S \times N \times \mathbb{Z}_{+} \times$ $\{0,1\}$, where $N=\{1, \ldots, n\}$ stands for the set of agents ordered accordingly, and $\mathbb{Z}_{+}$stands for the set of positive integers. That is, each agent announces a state, an agent, a positive integer, and raises a flag or not. Let the outcome function $g$ be such that:

(1) If everybody announces the same state $s$, then we have $g(m)=h(s)$.

(2) Suppose everybody but $i$ announces the state $s$, and $i$ announces the state $s^{\prime}$ and raises a flag, where the set of agents that raise a flag is $K$. Now, if $K \in \gamma\left(s, s^{\prime}\right)$ then $g(m)=h\left(s^{\prime}\right)$; if not, $g(m)=h(s)$.

(3) Otherwise, let $i$ be the agent with the minimum index among those who announce the highest integer. Let $j$ be the agent pointed at by agent $i$. Let $s^{\prime \prime}$ be the state announced by agent $j$. Then we have $g(m)=h\left(s^{\prime \prime}\right)$.

Now, consider the mechanism $(M, g)$. For each $u \in \mathcal{P}$ and $s \in E(\Gamma, u)$, consider any strategy profile $m$ such that each agent announces the state $s$. Since $s \in E(\Gamma, u)$, we have for each $s^{\prime} \neq$ $s$ with $h\left(s^{\prime}\right) \neq h(s)$ and coalition $K$, if $h\left(s^{\prime}\right) u_{K} h(s)$ then $K \notin \gamma\left(s, s^{\prime}\right)$. It follows that $m \in$ $\operatorname{SNE}(M, g, u)$.

Next, we show that for each $m \in S N E(M, g, u)$, we have $g(m) \in h(E(\Gamma, u))$. By contradiction, suppose there exists $m \in S N E(M, g, u)$ such that $g(m) \notin h(E(\Gamma, u))$. It means for each $s \in S$ with $g(m)=h(s)$, we have $s \notin E(\Gamma, u)$. It follows that there exist $s^{\prime} \in S$ and a coalition 
$K$ such that $K \in \gamma\left(s, s^{\prime}\right)$ and $h\left(s^{\prime}\right) u_{K} h(s)$. We will obtain the contradiction by showing that coalition $K$ can deviate to obtain $h\left(s^{\prime}\right)$.

Case 1: Suppose $m$ is as in (1). Let each $i \in K$ raise a flag and let one of them announce the state $s^{\prime}$. Since $K \in \gamma\left(s, s^{\prime}\right)$, the outcome is $h\left(s^{\prime}\right)$.

Case 2: Suppose $m$ is as in (2). Let $t$ stand for the state announced by everyone but agent $i$, who announces another state $w$. Let $L$ be the set of agents who raise a flag. Now there are two possibilities.

First, suppose that $w=s$ and $L \in \gamma(t, s)$. If coalition $K$ contains an agent $j$ who announces the state $s$, then $j$ can deviate to obtain $h\left(s^{\prime}\right)$ as follows: $j$ announces the highest integer, if $t \neq s^{\prime}$ then $j$ announces state $s^{\prime}$ and points at himself; if $t=s^{\prime}$ then $j$ announces a third state and points at one of the agents who announces $s^{\prime}$ (since $n \geq 3$, there is such an agent). If $K$ does not contain such an agent $j$, it follows that $K=\{i\}$. But now we have $L \in \gamma(t, s)$ and $\{i\} \in \gamma\left(s, s^{\prime}\right)$. If $t \neq s^{\prime}$ then it follows from CT that $L \in \gamma\left(t, s^{\prime}\right)$. Regardless of whether $t=s^{\prime}$ or not, if $j$ announces $s^{\prime}$ then the outcome is $h\left(s^{\prime}\right)$, and thus $j$ would deviate to announce $s^{\prime}$ instead of $s$.

Next, suppose $t=s$ and $L \notin \gamma(s, w)$. If coalition $K$ contains an agent $j$ who announces the state $s$, then $j$ can deviate to obtain $h\left(s^{\prime}\right)$ as follows: $j$ announces the highest integer, if $w \neq s^{\prime}$, then $j$ announces state $s^{\prime}$ and points at himself; if $w=s^{\prime}$, then $j$ announces a third state and points at agent $i$ who announces $s^{\prime}$. If coalition $K$ does not contain such an agent $j$, it follows that $K=\{i\}$. But now, we have $\{i\} \in \gamma\left(s, s^{\prime}\right)$. Since $i \in L$, it follows from CA that $L \in \gamma\left(s, s^{\prime}\right)$. Hence $i$ can obtain $h\left(s^{\prime}\right)$ by announcing $s^{\prime}$ instead of $w$.

Case 3: Suppose $m$ is as in (3). Now coalition $K$ can deviate to obtain $h\left(s^{\prime}\right)$ as follows: If no one has announced $s^{\prime}$, then an agent in $K$ announces the state $s^{\prime}$, the highest integer, and points at himself. If some agent $j$ has announced $s^{\prime}$ and $K \neq\{j\}$, then an agent in $K$ announces the highest integer, and points at $j$. If some agent $j$ has announced $s^{\prime}$ and $K=\{j\}$, then $j$ announces the state $s^{\prime}$, the highest integer, and points at himself. Thus, we reach the contradiction that $m \notin S N E(M, g, u)$.

\section{Implementation via code of rights}

A simple candidate for the state space of a rights structure is the set of alternatives. Suppose that the outcome function is the identity map. In this setting, the unique design object is the code of rights. In this section, we analyze this specific form of implementation via rights structures, which we call implementation via code of rights ( $\gamma$-implementation). We obtain the following definition for $\gamma$-equilibrium if we choose the alternative set $A$ as the state space and the identity function as the outcome function in $\Gamma$-equilibrium.

Definition 3. For each $u \in \mathcal{P}$, we say $a \in A$ is a $\gamma$-equilibrium at $u$ if for each $b \in A$, there is no coalition $K \in \gamma(a, b)$ with $b u_{K} a$.

An alternative $a$ is a $\gamma$-equilibrium at a given preference profile $u$, if there is no coalition $K$ such that $K$ is entitled to approve a change from $a$ to another alternative $b$, and each member of $K$ prefers $b$ to $a$. We denote $\gamma$-equilibria at preference profile $u$ by $E(\gamma, u)$.

Definition 4. An SCR $F$ is $\gamma$-implementable if there exists a code of rights $\gamma$ such that for each $u \in \mathcal{P}, F(u)=E(\gamma, u)$. 
We provide a full characterization of $\gamma$-implementable SCRs. It follows from Proposition 1 that an SCR should satisfy Maskin monotonicity to be $\gamma$-implementable. However, one can easily verify that Maskin monotonicity is not sufficient for $\gamma$-implementability. We introduce a new property that we call binary consistency. To introduce binary consistency, we need an auxiliary definition. Consider any $u \in \mathcal{P}$ and $a, b \in A$ such that $b$ is not Pareto dominated by $a$ at $u$. Let $u^{a b}$ stand for the preference profile obtained from $u$ in which $a$ and $b$ are now the two most preferred alternatives for all agents and their relative ranking is unchanged. That is, for each $c \in A \backslash\{a, b\}$ and $i \in N$, we have $a u_{i}^{a b} c$ and $b u_{i}^{a b} c$, and $\left\{i \in N: b u_{i} a\right\}=\left\{i \in N: b u_{i}^{a b} a\right\}$ holds. Next, we present the formal definition of binary consistency.

Definition 5. An SCR $F$ satisfies binary consistency if for each $u \in \mathcal{P}$ and $a \in A$, we have $a \in F(u)$ whenever for each $b \in A$ that is not Pareto dominated by $a$ at $u$, we have $a \in F\left(u^{a b}\right)$.

To interpret binary consistency, consider a preference profile $u$ and alternative $a$. Now, for each other alternative $b$, consider $u^{a b}$. Note that, by our choice of $u^{a b}$, the Pareto efficient alternatives at $u^{a b}$ are $a$ and $b$. Binary consistency requires that if $a$ is chosen at $u^{a b}$ for each alternative $b$, then $a$ should also be chosen at $u$. Intuitively, binary consistency tells that if $a$ is a winner at each binary comparison, then $a$ should be a winner when compared to all alternatives. We observe that binary consistency is satisfied by all of the examples in Section 3.1, except the tops rule (Example 5). To see how the tops rule fails to satisfy binary consistency, consider a preference profile $u$ and an alternative $a$ such that $a$ is neither Pareto dominated nor top ranked at $u$. Since $a$ is not Pareto dominated, for each other alternative $b$ we have $a \in F\left(u^{a b}\right)$, but $a \notin F(u)$. In our next proposition, we show that monotonicity strengthened by binary consistency characterizes implementability via codes of rights. ${ }^{18}$

Proposition 5. An SCR F is $\gamma$-implementable if and only if $F$ satisfies Maskin monotonicity and binary consistency.

Proof. $(\Rightarrow)$ Suppose that $F$ is a $\gamma$-implementable SCR. It follows from Proposition 1 that $F$ is monotonic. To see how $F$ satisfies binary consistency, consider any profile $u \in \mathcal{P}$, and let $a \in A$ be such that for each $b \in A$ that is not Pareto dominated by $a$ at $u$, we have $a \in F\left(u^{a b}\right)$. We show that $a \in F(u)$. Suppose not, i.e. $a \notin F(u)$. Since $F(u)=E(\gamma, u)$, there exist an alternative $b \neq a$ and a coalition $K \in \gamma(a, b)$ such that $b u_{K} a$. Since $\left\{i \in N: b u_{i} a\right\}=\left\{i \in N: b u_{i}^{a b} a\right\}$, we get $b u_{K}^{a b} a$. It follows that $a \notin E\left(\gamma, u^{a b}\right)$. Hence we obtain the contradiction $a \notin F\left(u^{a b}\right)$.

$(\Leftarrow)$ Let $F$ be an SCR that satisfies Maskin monotonicity and binary consistency. For each pair $a, b \in A$, a coalition $K$ is $(a, b)$-blocking if for each $u \in \mathcal{P}$, we have $a \notin F(u)$ whenever for each $i \in K, b u_{i} a$. Now, design the code of rights, $\gamma$, such that for each $a, b \in A$, a coalition $K \in \gamma(a, b)$ if and only if $K$ is $(a, b)$-blocking. Next, we show that $\gamma$ implements $F$. Let $u \in \mathcal{P}$, we first show that if $a \in F(u)$, then $a \in E(\gamma, u)$. To see this, since $a \in F(u)$, there is no $(a, b)$-blocking coalition with $b u_{K} a$. It follows that $a \in E(\gamma, u)$.

Next, we show that if $a \in E(\gamma, u)$, then $a \in F(u)$. Suppose not, i.e. $a \notin F(u)$. Since $F$ satisfies binary consistency, there should exist $b \in A$, not Pareto dominated by $a$ at $u$, such that $a \notin$ $F\left(u^{a b}\right)$. Let $K=\left\{i \in N: b u_{i} a\right\}$. Next, we argue that $K$ is an $(a, b)$-blocking coalition. To see this, let $u^{\prime}$ be any preference profile such that for each $i \in K, b u_{i}^{\prime} a$. We have to show that

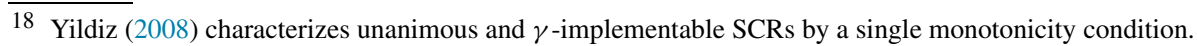


$a \notin F\left(u^{\prime}\right)$. Suppose not, i.e. $a \in F\left(u^{\prime}\right)$. Then, by the construction of $u^{a b}, K=\left\{i \in N: b u_{i}^{a b} a\right\}$. Moreover, for each $c \in A \backslash\{a, b\}$ and $i \in N$, we have $a u_{i}^{a b} c$. It follows from monotonicity that $a \in F\left(u^{a b}\right)$, leading to a contradiction. Hence, we conclude that $K$ is an $(a, b)$-blocking coalition. Since $b u_{K} a$, we have $a \notin E(\gamma, u)$. But this contradicts our initial supposition. It follows that if $a \in E(\gamma, u)$, then $a \in F(u)$.

\section{Deviation-constrained mechanisms}

In this section we formulate a strategic framework for implementation that turns out to be equivalent to implementability via rights structures in the presence of at least three agents. We call the design object of this environment a deviation-constrained mechanism (dc-mechanism). In a classical mechanism, an agent can deviate from a joint strategy by choosing any strategy from his strategy set, as he is independent of the joint strategy from which the deviation is to be made. On this front, a dc-mechanism is different from a classical mechanism. Namely, deviation strategies of each agent are constrained depending on the joint strategy from which the deviation is to be made.

We observe that specific forms of deviation constraints have been considered previously in the mechanism design literature. For example, Hurwicz et al. (1995) assume that an agent can claim to have any subset of his true endowment but cannot claim a larger set of endowments in a pure exchange economy setting. More recently, Glazer and Rubinstein (2012) present a persuasion situation as a leader-follower relation. In their model, the persuasion rule and its frame is determined by the leader. As in a dc-mechanism, the strategies that the follower can choose vary depending on how the leader frames the persuasion rule.

From a bounded rationality perspective, implementation via deviation-constrained mechanisms proposes a general framework that allows the designer to additionally structure the actions that each agent pays attention whenever he considers a deviation from a given joint action. It can be too general and unrealistic to assume that the designer can shape the actions that grab the attention of each agent in any arbitrary way. However, this exercise highlights a general connection between our $\Gamma$-implementability and implementation with agents who may exhibit limited attention - in the vein of Masatlioglu et al. (2012) - to their available strategies.

Formally, a dc-mechanism is a triple $(M, \mathcal{D}, g)$. As usual, the joint strategy space $M=$ $\Pi_{i \in N} M_{i}$, where $M_{i}$ stands for the strategy set of agent $i$. The outcome function $g$ maps every joint strategy to an alternative, i.e. $g: M \rightarrow A$. For each agent $i$, a constraint function $\mathcal{D}_{i}$ maps each joint strategy $m$ to a subset of $M_{i}$, i.e. $\mathcal{D}_{i}: M \rightarrow M_{i}$. In a dc-mechanism, if an agent $i$ would deviate from strategy $m$, he is constrained to choosing his strategy from $\mathcal{D}_{i}(m)$. Given a preference profile $u$, a joint strategy $m$ is an equilibrium of the dc-mechanism $(M, \mathcal{D}, g)$ at $u$ if for each $i \in N$ and $m_{i}^{\prime} \in \mathcal{D}_{i}(m), g(m) u_{i} g\left(m_{i}^{\prime}, m_{-i}\right)$. We denote the equilibria of $(M, \mathcal{D}, g)$ at $u$ by $\mathbf{E}(M, \mathcal{D}, g, u){ }^{19}$

Proposition 6. In the presence of at least three agents, an SCR $F$ is $\Gamma$-implementable if and only if there exists a dc-mechanism $(M, \mathcal{D}, g)$ such that for each $u \in \mathcal{P}, F(u)=g(\mathbf{E}(M, \mathcal{D}, g, u))$.

\footnotetext{
19 The notion of social equilibria introduced by Debreu (1952) is similar to our equilibrium notion. Debreu analyzes a strategic environment, where the strategy choice of an agent is not entirely free but the joint strategy of the other agents determines the subset to which the first agent's strategy is restricted. Our formulation differs from this, since given a joint strategy, the deviation strategies of an agent are restricted not only by the strategies of the others, but also by his own strategy.
} 
Proof. $(\Leftarrow)$ This part follows from reasoning similar to the one we used in the proof of Proposition 3. $(\Rightarrow)$ Suppose $F$ is implementable via a rights structure $(S, h, \gamma)$. By the virtue of Proposition 1, we can w.l.o.g. assume that this rights structure is individual based. Now let $\Sigma$ denote the collection of all finite sequences consisting of distinct states $\left(s_{1}, \ldots, s_{k}\right)$ from $S$. For each $i \in N$, let $M_{i}=\Sigma \times N \times \mathbb{Z}_{+}$, where $N=\{1, \ldots, n\}$ stands for the set of agents ordered accordingly and $\mathbb{Z}_{+}$stands for the set of positive integers. First, let us specify the deviation constraints. For each $m \in M$,

(DC1) If everybody announces the same single state then each agent can deviate to a strategy where he announces a single state, i.e. for each $i \in N, \mathcal{D}_{i}(m)=S \times N \times \mathbb{Z}_{+}$.

(DC2) If everybody but $i$ announces the same single state and $i$ announces another single state, then $i$ can deviate to any strategy, i.e. $\mathcal{D}_{i}(m)=M_{i}$. Any other agent $j \neq i$, can deviate to a strategy where $j$ announces a single state, i.e. for each $j \in N \backslash\{i\}, \mathcal{D}_{j}(m)=S \times N \times \mathbb{Z}_{+}$.

We pose no deviation constraints other than DC1 and DC2, so for every other $m \in M$ and for each $i \in N, \mathcal{D}_{i}(m)=M_{i}$. We define the outcome function $g: M \rightarrow A$ such that:

(1) If everybody announces the same single state $s$, then we have $g(m)=h(s)$.

(2) If everybody but $i$ announces the single state $s$ and $i$ announces the state array $\left\{s_{1}, \ldots, s_{k}\right\}$ such that $\{i\} \in \gamma\left(s, s_{1}\right) \cap \gamma\left(s_{1}, s_{2}\right) \cdots \cap \gamma\left(s_{k-1}, s_{k}\right)$, then $g(m)=h\left(s_{k}\right)$. If $\{i\} \notin \gamma\left(s, s_{1}\right) \cap$ $\gamma\left(s_{1}, s_{2}\right) \cdots \cap \gamma\left(s_{k-1}, s_{k}\right)$, then $g(m)=h(s)$.

(3) Otherwise, let $i$ be the agent with the minimum index among those who announce the highest integer. Let agent $j$ be the agent whom is indicated by agent $i$. Let $s_{k}$ be the last state in the state array announced by agent $j$. We then have $g(m)=h\left(s_{k}\right)$.

Next, consider the dc-mechanism $(M, \mathcal{D}, g)$. For each $u \in \mathcal{P}$ and $s \in E(\Gamma, u)$, consider any strategy profile $m$ such that for every $i \in N, m_{i, 1}=s$. Recall that each $i \in N$ can deviate from $m$ only by announcing a single state, i.e. $D_{i}(m)=S \times N \times \mathbb{Z}_{+}$. Since $s \in E(\Gamma, u)$, we have for every $s^{\prime} \neq s$ with $h\left(s^{\prime}\right) \neq h(s)$ and $i \in N$, if $h(s) u_{i} h\left(s^{\prime}\right)$ then $\{i\} \notin \gamma\left(s, s^{\prime}\right)$. It follows that $m \in \mathbf{E}(M, D, g, u)$.

Next, we show that for each $m \in \mathbf{E}(M, \mathcal{D}, g, u)$, we have $g(m) \in h(E(\Gamma, u))$. We show this through reasoning similar to that in the proof of Proposition 3, and thus we do not replicate it here. Yet, there is only one case where we use a different line of reasoning to obtain the contradiction - the case where we used the IT condition.

Case 2: Suppose $g(m)$ is realized by condition (2). If $m_{i, 1}=s_{1}, \ldots, s_{k}, s$ and for every $j \neq i$, $m(j, 1)=\bar{s}$. Since $m \in \mathbf{E}(M, D, g, u)$ with $g(m)=h(s)$, we have $\{i\} \in \gamma\left(\bar{s}, s_{1}\right) \cap \cdots \cap \gamma\left(s_{k}, s\right)$. Moreover, we know that $\{i\} \in \gamma\left(s, s^{\prime}\right)$. Now, let $m_{i}^{\prime}$ be such that $m_{i, 1}^{\prime}=s_{1}, \ldots, s_{k}, s^{\prime}$. Notice that by (2) $m_{i}^{\prime} \in \mathcal{D}_{i}(m)$, and we have $g\left(m_{i}^{\prime}, m_{-i}\right)=h\left(s^{\prime}\right)$.

Proposition 6 shows that the additional freedom of designing the deviation constraints does not bring much in terms of implementability. This is because deviation constraints should be both stringent enough to keep a particular strategy when it is an equilibrium at a preference profile, and also permissive enough to eliminate the same strategy when it is not an equilibrium at another preference profile. Some recent research on mechanism design with evidence ${ }^{20}$ and mechanism design with boundedly rational agents is related to our dc-mechanisms. The most

20 Most of this literature concerns partial-implementation with evidence. An early reference is Green and Laffont (1986); recent studies include Bull and Watson (2004, 2007), Deneckere and Severinov (2008), and Glazer and Rubinstein (2004). 
relevant ones are Kartik and Tercieux (2012) and de Clippel (2014). We discuss these in the rest of this section.

Kartik and Tercieux (2012) generalize the Nash implementation problem by assuming that agents can additionally provide some verifiable evidence about the state of the world. A piece of evidence can be thought of as a discriminatory signal regarding the preference profile in the society. In this setting, the designer can specify the outcome function contingent both on the joint strategy profile and the reported evidence profile. In contrast, in an dc-mechanism the designer can only specify the set of strategies that an agent can use to deviate from a given strategy profile. These "allowed" deviations are contingent on strategy profiles but independent of the society's true preference profile. Therefore, it does not act as a signaling device, as in the case of implementation with evidence. This basic difference also reveals itself in terms of the characterization results, in that Example 1 of Kartik and Tercieux (2012) shows that Maskin-monotonicity is not necessary for implementation with evidence. This example shows that if the designer has the power to design the state dependent evidence structure, then any SCR can be easily implemented.

In a recent study, de Clippel (2014) provides a general framework, called behavioral implementation, that incorporates boundedly rational choice theories into implementation problem. In particular, de Clippel investigates the implementation problem when agents exhibit limited attention. Our analysis in this section differs from the model and analysis of de Clippel in two main ways. First, de Clippel assumes that a social choice rule aggregates a choice function profile. In contrast, we assume, as usual, that a social choice rule aggregates a preference profile. Secondly, in both models, given a mechanism, each agent can be boundedly rational in choosing an action. However, in our model, we assume that the attention filter of the agents is also subject to design. Thus, we can implement social choice rules that can be implemented neither in the classical setup, nor in de Clippel's setup.

\section{Conclusion}

In this paper, we have dealt with a novel framework for implementation, which explicitly takes the rights structure introduced by Sertel (2001) as its point of departure. Our results indicate that implementation via rights structures - referred to as $\Gamma$-implementation-is both transparent and general, since all image monotonic social choice rules are $\Gamma$-implementable via the canonical rights structure, which is free of integer game like constructs.

The notion of $\Gamma$-implementation also yields novel characterizations of Nash and strong Nash implementability via a mechanism. One possible reason for the transparency and generality of $\Gamma$-implementation is that we leave unspecified the details of the agents' interaction in reaching a $\Gamma$-equilibrium. In this study, we addressed this problem on two different grounds, one of which is concerned with convergence from a nonequilibrium state into an equilibrium state. The second ground involves finding the precise strategic counterpart of $\Gamma$-implementation in terms of mechanisms.

In summary, the achievements in this study can be summarized as the introduction of a novel tool for implementation of social choice rules in terms of rights structures, together with its connections to classical implementation via mechanisms. Although our study covers a rather extensive treatment of the subject, we can hardly claim that it is exhaustive, as it leads to a wide variety of open problems yet to be solved. 


\section{References}

Abreu, Dilip, Matsushima, Hitoshi, 1992. Virtual implementation in iteratively undominated strategies: complete information. Econometrica 60 (5), 993-1008.

Abreu, Dilip, Sen, Arunava, 1991. Virtual implementation in Nash equilibrium. Econometrica 59 (4), 997-1021.

Acemoglu, Daron, Egorov, Georgy, Sonin, Konstantin, 2012. Dynamics and stability of constitutions, coalitions, and clubs. Am. Econ. Rev. 102 (4), 1446-1476.

Arrow, Kenneth J., 1967. Values and collective decision-making. In: Philosophy, Politics, and Society, third series. Basil Blackwell, Oxford, pp. 215-332.

Aumann, J. Robert, Maschler, Michael, 1964. The bargaining set for cooperative games. In: Advances in Game Theory. Princeton University Press, Princeton, NJ.

Barbera, Salvador, Jackson, Matthew O., 2004. Choosing how to choose: self-stable majority rules and constitutions. Q. J. Econ., 1011-1048.

Bull, Jesse, Watson, Joel, 2004. Evidence disclosure and verifiability. J. Econ. Theory 118 (1), 1-31.

Bull, Jesse, Watson, Joel, 2007. Hard evidence and mechanism design. Games Econ. Behav. 58 (1), 75-93.

Cabrales, Antonio, Serrano, Roberto, 2011. Implementation in adaptive better-response dynamics: towards a general theory of bounded rationality in mechanisms. Games Econ. Behav. 73 (2), 360-374.

Chwe, Michael Suk-Young, 1994. Farsighted coalitional stability. J. Econ. Theory 63, 229-325.

Danilov, Vladimir, 1992. Implementation via Nash equilibria. Econometrica 60 (1), 43-56.

de Clippel, Geoffroy, 2014. Behavioral implementation. Am. Econ. Rev. 104 (10), 2975-3002.

Debreu, Gerard, 1952. A social equilibrium existence theorem. Proc. Natl. Acad. Sci. USA 38, 886-893.

Deneckere, Raymond, Severinov, Sergei, 2008. Mechanism design with partial state verifiability. Games Econ. Behav. 64 (2), 487-513.

Dutta, Bhaskar, Sen, Arunava, 1991. A necessary and sufficient condition for two person Nash implementation. Rev. Econ. Stud. 58 (1), 121-128.

Dutta, Bhaskar, Sen, Arunava, 2012. Nash implementation with partially honest individuals. Games Econ. Behav. 74, 154-169.

Glazer, Jacob, Rubinstein, Ariel, 2012. A model of persuasion with boundedly rational agents. J. Polit. Econ. 120 (6), 1057-1082.

Glazer, Jacob, Rubinstein, Ariel, 2004. On optimal rules of persuasion. Econometrica 72 (6), 1715-1736.

Green, Jerry R., Laffont, Jean-Jacques, 1986. Partially verifiable information and mechanism design. Rev. Econ. Stud. 53 (3), 447-456.

Greenberg, Joseph, 1990. The Theory of Social Situations: An Alternative Game-Theoretic Approach. Cambridge Univ. Press, Cambridge.

Hurwicz, Leonid, 1972. On informationally decentralized systems. In: Decision and Organization. North Holland, Amsterdam.

Hurwicz, Leonid, Maskin, Eric, Postlewaite, Andrew, 1995. Feasible Nash implementation of social choice rules when the designer does not know endowments or production sets. In: The Economics of Informational Decentralization: Complexity, Efficiency, and Stability. Springer, pp. 367-433.

Jackson, Matthew O., 1992. Implementation in undominated strategies: a look at bounded mechanisms. Rev. Econ. Stud. 59 (4), 257-775.

Kartik, Navin, Tercieux, Olivier, 2012. Implementation with evidence. Theor. Econ. 7 (2), 323-355.

Koray, Semih, 2000. Self-selective social choice functions verify arrow and Gibbard-Satterthwaite theorems. Econometrica 68 (4), 981-996.

Masatlioglu, Yusufcan, Nakajima, Daisuke, Ozbay, Erkut Y., 2012. Revealed attention. Am. Econ. Rev. 102 (5), 2183-2205.

Maskin, Eric, 1985. The theory of implementation in Nash equilibrium: a survey. In: Hurwicz, L., Scheidler, D., Sonnenschein, H. (Eds.), Social Goals and Social Organization. Cambridge University Press.

McKelvey, Richard D., 1989. Game forms for Nash implementation of general social choice correspondences. Soc. Choice Welf. 6, 139-156.

McQuillin, Ben, Sugden, Robert, 2011. The representation of alienable and inalienable rights: games in transition function form. Soc. Choice Welf. 37, 683-706.

Moore, John, Repullo, Rafael, 1988. Subgame perfect implementation. Econometrica 56 (5), 1191-1220.

Moulin, Herve, Peleg, Bezalel, 1982. Cores of effectivity functions and implementation theory. J. Math. Econ. 10, $115-145$. 
Palfrey, Thomas R., Srivastava, Sanjay, 1991. Nash implementation using undominated strategies. Econometrica 59 (2), 479-501.

Peleg, Bezalel, Winter, Eyal, 2002. Constitutional implementation. Rev. Econ. Des. 7, 187-204.

Rosenthal, Robert W., 1972. Cooperative games in effectiveness form. J. Econ. Theory 5, 88-101.

Rubinstein, Ariel, 1980. Stability of decision systems under majority rule. J. Econ. Theory 23, 150-159.

Sen, Amartya K., 1970. The impossibility of a Paretian liberal. J. Polit. Econ. 78, 152-157.

Sertel, R. Murat, 2001. Designing rights: invisible hand theorems, covering and membership. Mimeo. Bogazici University.

von Neumann, John, Morgenstern, Oskar, 1953. Theory of Games and Economic Behavior. Princeton Univ. Press, Princeton, NJ.

Yildiz, Kemal, 2008. Implementation via Codes of Rights. Master's thesis. Bilkent University. 